\title{
Triterpene Glycosides from the Whole Plant of Anemone hupehensis var. japonica and Their Cytotoxic Activity
}

\author{
Akihito Yokosuka, ${ }^{*, a}$ Tomoe Sano, ${ }^{a}$ Ken Hashimoto, ${ }^{b}$ Hiroshi Sakagami, ${ }^{b}$ and Yoshihiro Mimaki ${ }^{*}, a$ \\ ${ }^{a}$ Laboratory of Medicinal Pharmacognosy, School of Pharmacy, Tokyo University of Pharmacy and Life Sciences; 1432-1 \\ Horinouchi, Hachioji, Tokyo 192-0392, Japan: and ${ }^{b}$ Department of Diagnostic and Therapeutic Sciences, School of \\ Dentistry, Meikai University; 1-1 Keyakidai, Sakado, Saitama 350-0283, Japan.
}

Received August 20, 2009; accepted September 28, 2009; published online September 29, 2009

Three new triterpene glycosides (1-3), together with eight known triterpene glycosides (4-11), were isolated from the whole plant of Anemone hupehensis var. japonica (Ranunculaceae). The structures of the new compounds were determined on the basis of spectroscopic analysis and the results of hydrolytic cleavage experiments. The isolated compounds were evaluated for their cytotoxic activities against HL-60 human leukemia cells, HSC-2 human oral squamous carcinoma cells, HSC-4 human oral squamous carcinoma cells, and A549 human lung adenocarcinoma cells.

Key words triterpene glycoside; Anemone hupehensis var. japonica; Ranunculaceae; cytotoxic activity

Anemone hupehensis LEM. var. japonica (THunB.) Bowles et STEARN is a perennial plant belonging to the family Ranunculaceae and is distributed throughout Japan and China. ${ }^{1)}$ Its roots have been used in traditional Chinese medicine for the treatment of fever. ${ }^{2)}$ Although the triterpene glycosides hupehensis saponin $\mathrm{A}-\mathrm{G}$ were isolated from A. hupehensis, ${ }^{3)}$ there have been no reports concerning the secondary metabolites of A. hupehensis var. japonica. Therefore, in this study, we conducted a phytochemical screening of the whole plant. As a result, three new triterpene glycosides $(\mathbf{1}-\mathbf{3})$ and eight known triterpene glycosides (4-11) were isolated. Here, we describe the isolation and structural elucidation of the three new triterpene glycosides $(\mathbf{1}-\mathbf{3})$ on the basis of spectroscopic analysis, including various two-dimensional (2D) NMR spectroscopic studies, and the results of hydrolytic cleavage experiments. The cytotoxic activities of the isolated compounds against HL-60 human leukemia cells, A549 human lung adenocarcinoma cells, HSC-2 human oral squamous carcinoma cells, and HSC-4 human oral squamous carcinoma cells are also reported.
The whole plant of $A$. hupehensis var. japonica $(3.3 \mathrm{~kg})$ was extracted with hot $\mathrm{MeOH}$. After removal of solvent, the $\mathrm{MeOH}$ extract was passed through a porous-polymer polystyrene resin (Diaion HP-20) column successively eluted with $30 \% \mathrm{MeOH}, 50 \% \mathrm{MeOH}, \mathrm{MeOH}$, EtOH, and EtOAc. The $\mathrm{MeOH}$ eluted fraction was repeatedly subjected to silica gel and octadecylsilanized (ODS) silica gel column chromatography to afford compounds 1-11. Compounds 4-11 were identified by their physical and spectroscopic data as $3 \beta$ - $[(O-\beta$-D-ribopyranosyl- $(1 \rightarrow 3)-O$ - $\alpha$-L-rhamnopyranosyl$(1 \rightarrow 2)-\alpha$-L-arabinopyranosyl)oxy]olean-12-en-28-oic acid (prosapogenin $\left.\mathrm{CP} 4,4),{ }^{4}\right) 3 \beta$-[( $O$ - $\beta$-D-ribopyranosyl- $(1 \rightarrow 3)$ $O$ - $\alpha$-L-rhamnopyranosyl-( $1 \rightarrow 2)$ - $\beta$-D-xylopyranosyl)oxy]olean12-en-28-oic acid (huzhangoside A, 5), ${ }^{4)}$ 23-hydroxy-3 $\beta$ $[(O-\beta$-D-ribopyranosyl- $(1 \rightarrow 3)-O$ - $\alpha$-L-rhamnopyranosyl$(1 \rightarrow 2)-\alpha$-L-arabinopyranosyl)oxy]olean-12-en-28-oic acid (prosapogenin CP6, 6), ${ }^{4}$ ) olean-12-en-28-oic acid $O-\alpha$-Lrhamnopyranosyl- $(1 \rightarrow 4)-O$ - $\beta$-D-glucopyranosyl- $(1 \rightarrow 6)-\beta$-Dglucopyranosyl ester (cussonoside $\mathrm{B}, 7),{ }^{5)} 3 \beta$-[(O- $\beta$-D-ribopyranosyl-( $1 \rightarrow 3)-O-\alpha$-L-rhamnopyranosyl- $(1 \rightarrow 2)-\alpha$-L-

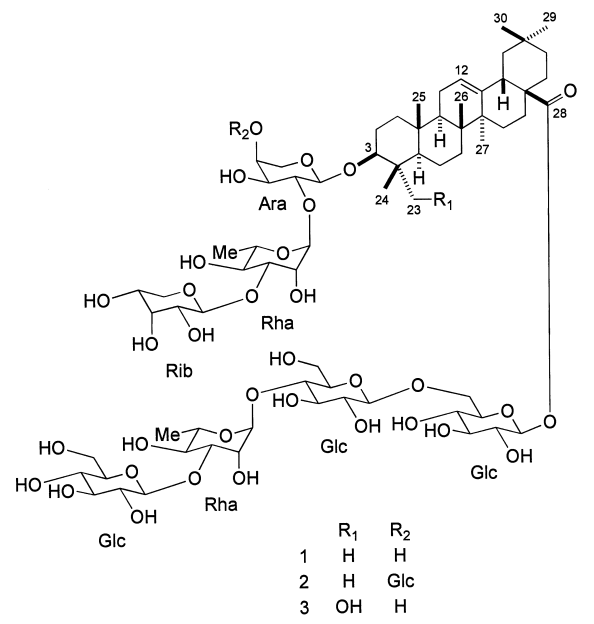

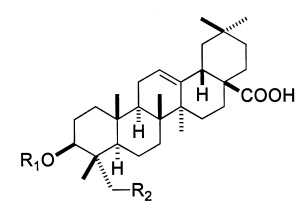

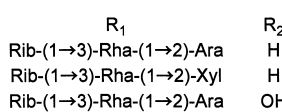

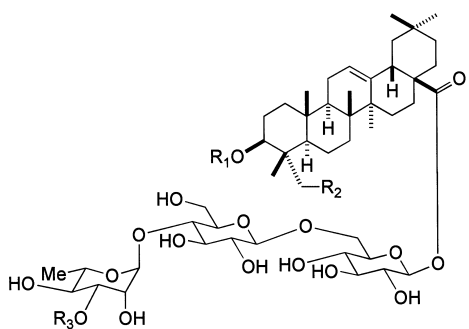
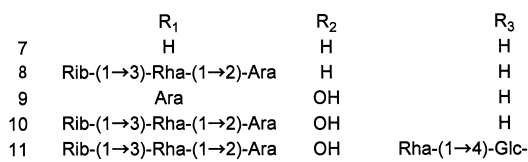

Rha- $(1 \rightarrow 4)-G l c-(1 \rightarrow 6)-G l c$

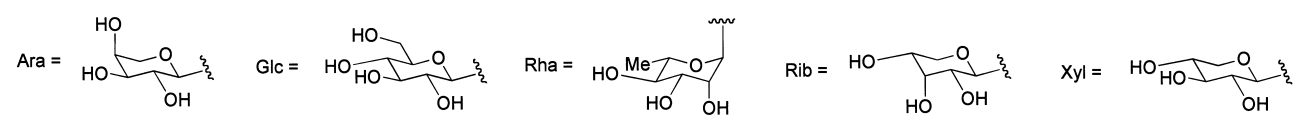


arabinopyranosyl)oxy]olean-12-en-28-oic acid $O$ - $\alpha$-L-rhamnopyranosyl-( $1 \rightarrow 4)-O$ - $\beta$-D-glucopyranosyl- $(1 \rightarrow 6)-\beta$-Dglucopyranosyl ester (huzhangoside B, 8), ${ }^{4)} 3 \beta$ - $[(\alpha$-L-arabinopyranosyl)oxy]-23-hydroxyolean-12-en-28-oic acid $O$ - $\alpha$ L-rhamnopyranosyl-( $1 \rightarrow 4)-O$ - $\beta$-D-glucopyranosyl- $(1 \rightarrow 6)-\beta$ D-glucopyranosyl ester (9), ${ }^{6)} 23$-hydroxy-3 $\beta$-[( $O$ - $\beta$-D-ribopyranosyl-( $1 \rightarrow 3)$ - $O$ - $\alpha$-L-rhamnopyranosyl- $(1 \rightarrow 2)-\alpha$-L-arabinopyranosyl)oxy]olean-12-en-28-oic acid $O$ - $\alpha$-L-rhamnopyranosyl-( $1 \rightarrow 4)-O$ - $\beta$-D-glucopyranosyl- $(1 \rightarrow 6)$ - $\beta$-D-glucopyranosyl ester (huzhangoside $\left.\mathrm{D}, 10),{ }^{4}\right) 23$-hydroxy $3 \beta$-[(O- $\beta$ D-ribopyranosyl-( $1 \rightarrow 3)$ - $O$ - $\alpha$-L-rhamnopyranosyl- $(1 \rightarrow 2)$ $\alpha$-L-arabinopyranosyl)oxy]olean-12-en-28-oic acid $O$ - $\alpha$-Lrhamnopyranosyl- $(1 \rightarrow 4)-O$ - $\beta$-D-glucopyranosyl- $(1 \rightarrow 6)-\beta$-Dglucopyranosyl- $(1 \rightarrow 3)-O$ - $\alpha$-L-rhamnopyranosyl- $(1 \rightarrow 4)-O-\beta$ D-glucopyranosyl-( $1 \rightarrow 6)$ - $\beta$-D-glucopyranosyl ester (hupehensis saponin F, 11), ${ }^{7)}$ respectively.

Compound 1 was obtained as an amorphous solid and showed an accurate $[\mathrm{M}+\mathrm{Na}]^{+}$ion at $m / z \quad 1521.7109$ in the high resolution (HR)-electrospray ionization (ESI)-time of flight (TOF)-MS, corresponding to the empirical molecular formula $\mathrm{C}_{70} \mathrm{H}_{114} \mathrm{O}_{34}$. The IR spectrum of 1 was suggestive of a glycoside $\left(3376,1064 \mathrm{~cm}^{-1}\right)$ and indicated the presence of a carbonyl group $\left(1740 \mathrm{~cm}^{-1}\right)$ in the molecule. The ${ }^{1} \mathrm{H}-\mathrm{NMR}$ spectrum of 1 contained signals for seven quaternary methyl groups at $\delta 1.28,1.23,1.13,1.06,0.89,0.87$, and 0.85 (each $\mathrm{s})$, an olefinic proton at $\delta 5.39$ (brs), and seven anomeric protons at $\delta 6.27$ (br s), $6.22(\mathrm{~d}, J=8.0 \mathrm{~Hz}), 5.93$ (d, $J=4.4$ $\mathrm{Hz}$ ), 5.84 (br s), 5.34 (d, $J=7.8 \mathrm{~Hz}), 4.90$ (d, $J=7.9 \mathrm{~Hz}$ ), and $4.82(\mathrm{~d}, J=6.0 \mathrm{~Hz})$, and the methyl groups of two 6-deoxyhexopyranosyl moieties at $\delta 1.63(\mathrm{~d}, J=6.2 \mathrm{~Hz})$ and $1.52(\mathrm{~d}$, $J=6.1 \mathrm{~Hz}$ ). Acid hydrolysis of 1 with $1 \mathrm{M} \mathrm{HCl}$ in dioxane$\mathrm{H}_{2} \mathrm{O}(1: 1)$ yielded $3 \beta$-hydroxyolean-12-en-28-oic acid (1a, oleanolic acid), as well as L-rhamnose, D-ribose, L-arabinose, and D-glucose as the carbohydrate moieties. Identification of the monosaccharides, including their absolute configurations, was carried out by direct HPLC analysis of the hydrolysate. The C-3 oxymethine carbon and C-28 carbonyl carbon were observed at $\delta 88.7$ and 176.5 , respectively, in the ${ }^{13} \mathrm{C}-\mathrm{NMR}$ of $\mathbf{1}$, which suggests that $\mathbf{1}$ is a 3,28-bisdesmoside of oleanolic acid. In a comparison of the whole ${ }^{13} \mathrm{C}-\mathrm{NMR}$ spectrum of 1 with that of $3 \beta$-[( $O-\beta$-D-ribopyranosyl- $(1 \rightarrow 3)-O-\alpha$-Lrhamnopyranosyl-( $1 \rightarrow 2)-\alpha$-L-arabinopyranosyl)oxy]olean12-en-28-oic acid $\beta$-D-glucopyranosyl ester (clematichinenoside A) ${ }^{8}{ }^{8}$ the signals due to the aglycone moiety and the triglycoside residue linked to $\mathrm{C}-3$ of the aglycone were observed at almost the same positions for each of the compounds. However, differences were recognized in the tetraglycoside moiety attached to C-28 of the aglycone. The exact structures of the sugar moieties and their linkage positions to the aglycone were solved by detailed analysis of the one-dimensional (1D) totally correlated spectroscopy (TOCSY) and 2D NMR spectra. The ${ }^{1} \mathrm{H}-\mathrm{NMR}$ subspectra of individual monosaccharide units were obtained by using selective irradiation of easily identifiable anomeric proton signals, as well as non-overlapping proton signals in a series of 1D TOCSY experiments. ${ }^{9-11)}$ Subsequent analysis of the ${ }^{1} \mathrm{H}-{ }^{1} \mathrm{H}$ shift correlation spectroscopy (COSY) spectrum resulted in the sequential assignment of all the proton resonances due to the seven glycosyl units, including identification of their multiplet patterns and coupling constants (Table 1). The ${ }^{1} \mathrm{H}$-detected heteronuclear multiple-quantum coher- ence (HMQC) and ${ }^{1} \mathrm{H}$-detected heteronuclear single-quantum coherence (HSQC)-TOCSY spectra correlated the proton resonances with those of the corresponding one-bond coupled carbons, leading to unambiguous assignments of the carbon shifts. Comparison of the carbon chemical shifts thus assigned with those of reference methyl glycosides, ${ }^{12,13)}$ taking into account the known effects of $O$-glycosylation, indicated that 1 contains a $\mathrm{C}-2$ substituted $\alpha$-L-arabinopyranosyl moiety (Ara), two C-3 substituted $\alpha$-L-rhamnopyranosyl moieties (Rha, Rha'), a terminal $\beta$-D-ribopyranosyl moiety (Rib), a C-6 substituted $\beta$-D-glucopyranosyl moiety (Glc), a C-4 substituted $\beta$-D-glucopyranosyl moiety (Glc'), and a terminal $\beta$-D-glucopyranosyl moiety $\left(\mathrm{Glc}^{\prime \prime}\right)$. In the ${ }^{1} \mathrm{H}$-detected heteronuclear multiple-bond connectivity (HMBC) spectrum of 1, long-range correlations were observed between the anomeric proton $(\mathrm{H}-1)$ of Rib at $\delta_{\mathrm{H}} 5.93$ and $\mathrm{C}-3$ of Rha at $\delta_{\mathrm{C}} 81.2, \mathrm{H}-1$ of Rha at $\delta_{\mathrm{H}} 6.27$ and C-2 of Ara at $\delta_{\mathrm{C}} 75.3, \mathrm{H}-$ 1 of Ara at $\delta_{\mathrm{H}} 4.82$ and C-3 of the aglycone at $\delta_{\mathrm{C}} 88.7, \mathrm{H}-1$ of Glc" at $\delta_{\mathrm{H}} 5.34$ and C-3 of Rha' at $\delta_{\mathrm{C}} 83.8, \mathrm{H}-1$ of Rha' at $\delta_{\mathrm{H}} 5.84$ and C-4 of Glc ${ }^{\prime}$ at $\delta_{\mathrm{C}} 77.2, \mathrm{H}-1$ of Glc' at $\delta_{\mathrm{H}} 4.90$ and C- 6 of Glc at $\delta_{\mathrm{C}} 69.3$, and between H-1 of Glc at $\delta_{\mathrm{H}} 6.22$ and $\mathrm{C}-28$ of the aglycone at $\delta_{\mathrm{C}} 176.5$ (Fig. 1). Accordingly, the structure of $\mathbf{1}$ was formulated as $3 \beta$-[( $O-\beta$-D-ribopyranosyl-( $1 \rightarrow 3)$ - $O$ - $\alpha$-L-rhamnopyranosyl- $(1 \rightarrow 2)$ - $\alpha$-L-arabinopyranosyl)oxy]olean-12-en-28-oic acid $O$ - $\beta$-D-glucopyranosyl-( $1 \rightarrow 3)$ - $O$ - $\alpha$-L-rhamnopyranosyl-( $1 \rightarrow 4)-O$ - $\beta$-D-glucopyranosyl-( $1 \rightarrow 6)-\beta$-D-glucopyranosyl ester.

Compound 2 was found to have a molecular formula of $\mathrm{C}_{76} \mathrm{H}_{124} \mathrm{O}_{39}$ as determined by HR-ESI-TOF-MS analysis $(\mathrm{m} / \mathrm{z}$ $\left.1661.7778[\mathrm{M}+\mathrm{H}]^{+}\right)$. The deduced molecular weight of 2 was higher than that of $\mathbf{1}$, with the difference corresponding to a $\mathrm{C}_{6} \mathrm{H}_{10} \mathrm{O}_{5}$ unit. The ${ }^{1} \mathrm{H}-\mathrm{NMR}$ spectrum of $\mathbf{2}$ contained signals for eight anomeric protons at $\delta 6.32$ (brs), 6.26 (d, $J=$ $8.1 \mathrm{~Hz}$ ), 5.98 (d, $J=4.2 \mathrm{~Hz}$ ), 5.88 (br s), 5.38 (d, $J=7.8 \mathrm{~Hz}$ ), $5.14(\mathrm{~d}, J=7.8 \mathrm{~Hz}), 4.90(\mathrm{~d}, J=7.9 \mathrm{~Hz}), 4.72$ (d, $J=6.8 \mathrm{~Hz})$. On the basis of the spectral properties of 2 and the results of acid hydrolysis experiments, in which 2 yielded 1a, L-rhamnose, D-ribose, L-arabinose, and D-glucose, 2 was shown to be a triterpene glycoside closely related to $\mathbf{1}$; however, the

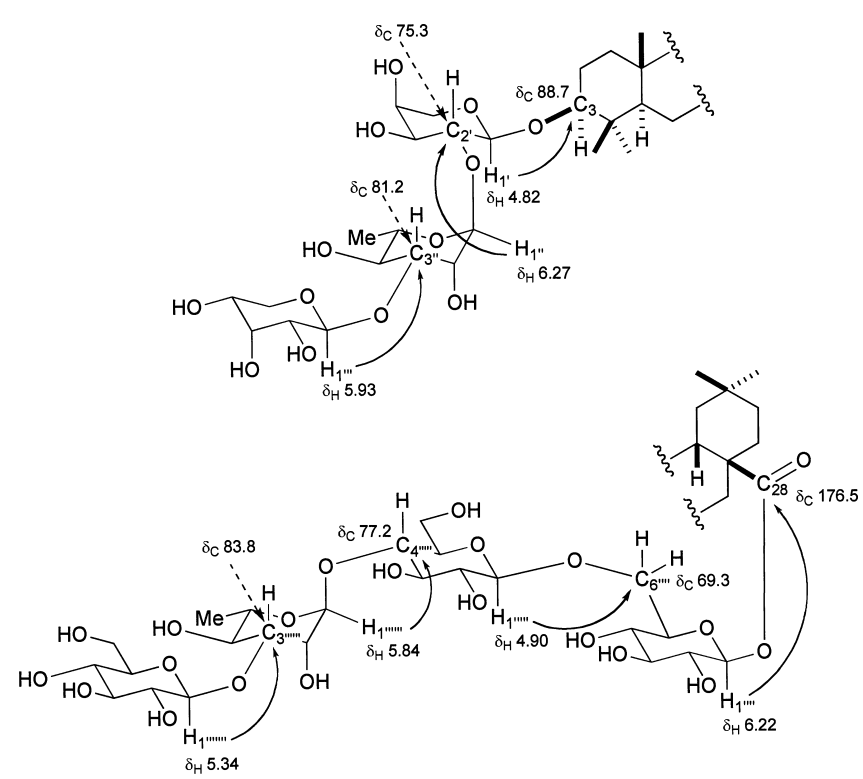

Fig. 1. HMBC Correlations of the Glycoside Moieties of $\mathbf{1}$ 
Table 1. ${ }^{1} \mathrm{H}$ - and ${ }^{13} \mathrm{C}-\mathrm{NMR}$ Chemical Shift Assignments of the Sugar Moieties for Compounds $\mathbf{1}-\mathbf{3}^{a}$ )

\begin{tabular}{|c|c|c|c|c|c|c|c|c|c|c|c|c|c|c|c|c|}
\hline \multicolumn{6}{|c|}{1} & \multicolumn{5}{|c|}{2} & \multicolumn{6}{|c|}{3} \\
\hline \multicolumn{3}{|c|}{ Position } & ${ }^{1} \mathrm{H}$ & $J(\mathrm{~Hz})$ & ${ }^{13} \mathrm{C}$ & \multicolumn{2}{|c|}{ Position } & ${ }^{1} \mathrm{H}$ & $J(\mathrm{~Hz})$ & ${ }^{13} \mathrm{C}$ & \multicolumn{3}{|c|}{ Position } & ${ }^{1} \mathrm{H}$ & $J(\mathrm{~Hz})$ & ${ }^{13} \mathrm{C}$ \\
\hline Ara & $1^{\prime}$ & & $4.82 \mathrm{~d}$ & 6.0 & 105.2 & Ara & $1^{\prime}$ & $4.72 \mathrm{~d}$ & 6.8 & 105.3 & Ara & $1^{\prime}$ & & $5.06 \mathrm{~d}$ & 6.6 & 105.0 \\
\hline & $2^{\prime}$ & & $4.57 \mathrm{dd}$ & $8.7,6.0$ & 75.3 & & $2^{\prime}$ & $4.51 \mathrm{dd}$ & $8.2,6.8$ & 75.8 & & $2^{\prime}$ & & $4.59 \mathrm{dd}$ & $8.1,6.6$ & 75.2 \\
\hline & $3^{\prime}$ & & $4.24 \mathrm{dd}$ & $8.7,4.7$ & 74.7 & & $3^{\prime}$ & 4.24 br d & 8.2 & 75.0 & & $3^{\prime}$ & & $4.02 \mathrm{dd}$ & $8.1,4.4$ & 75.4 \\
\hline & $4^{\prime}$ & & $4.21 \mathrm{~m}$ & & 69.4 & & $4^{\prime}$ & $4.23 \mathrm{~m}$ & & 80.4 & & $4^{\prime}$ & & $4.12 \mathrm{~m}$ & & 69.8 \\
\hline & $5^{\prime}$ & $\mathrm{a}$ & 4.29 br d & 11.0 & 65.7 & & $5^{\prime}$ & 4.38 br d & 10.7 & 65.5 & & $5^{\prime}$ & $\mathrm{a}$ & $4.25 \mathrm{dd}$ & $9.7,2.9$ & 66.4 \\
\hline & & $\mathrm{b}$ & 3.80 br d & 11.0 & & & $\mathrm{~b}$ & 3.75 br d & 10.7 & & & & $\mathrm{~b}$ & 3.67 br d & 10.9 & \\
\hline Rha & $1^{\prime \prime}$ & & 6.27 br s & & 101.4 & Rha & $1^{\prime \prime}$ & $6.32 \mathrm{br} \mathrm{s}$ & & 101.4 & Rha & $1^{\prime \prime}$ & & 6.36 br s & & 101.3 \\
\hline & $2^{\prime \prime}$ & & 4.89 br d & 3.7 & 72.0 & & $2^{\prime \prime}$ & 4.93 br d & 2.8 & 72.0 & & $2^{\prime \prime}$ & & 4.90 br d & 2.9 & 72.0 \\
\hline & $3^{\prime \prime}$ & & $4.72 \mathrm{dd}$ & $9.7,3.7$ & 81.2 & & $3^{\prime \prime}$ & $4.75 \mathrm{dd}$ & $9.6,2.8$ & 81.1 & & $3^{\prime \prime}$ & & $4.77 \mathrm{dd}$ & $9.6,2.9$ & 81.2 \\
\hline & $4^{\prime \prime}$ & & $4.42 \mathrm{dd}$ & $9.7,9.7$ & 72.8 & & $4^{\prime \prime}$ & $4.46 \mathrm{dd}$ & $9.6,9.6$ & 72.9 & & $4^{\prime \prime}$ & & $4.43 \mathrm{dd}$ & $9.6,9.6$ & 72.9 \\
\hline & $5^{\prime \prime}$ & & $4.61 \mathrm{dq}$ & $9.7,6.1$ & 69.8 & & $5^{\prime \prime}$ & $4.66 \mathrm{dq}$ & $9.6,6.1$ & 69.9 & & $5^{\prime \prime}$ & & $4.71 \mathrm{dq}$ & $9.6,6.2$ & 69.8 \\
\hline & $6^{\prime \prime}$ & & $1.52 \mathrm{~d}$ & 6.1 & 18.4 & & $6^{\prime \prime}$ & $1.58 \mathrm{~d}$ & 6.1 & 18.6 & & $6^{\prime \prime}$ & & $1.55 \mathrm{~d}$ & 6.2 & 18.4 \\
\hline Rib & $1^{\prime \prime \prime}$ & & $5.93 \mathrm{~d}$ & 4.4 & 104.6 & Rib & $1^{\prime \prime \prime}$ & $5.98 \mathrm{~d}$ & 4.2 & 104.8 & Rib & $1^{\prime \prime \prime}$ & & $5.96 \mathrm{~d}$ & 4.4 & 104.7 \\
\hline & $2^{\prime \prime \prime}$ & & $4.29 \mathrm{~m}$ & & 72.8 & & $2^{\prime \prime \prime}$ & $4.33 \mathrm{~m}$ & & 72.8 & & $2^{\prime \prime \prime}$ & & $4.31 \mathrm{~m}$ & & 72.8 \\
\hline & $3^{\prime \prime \prime}$ & & $4.51 \mathrm{~m}$ & & 70.2 & & $3^{\prime \prime \prime}$ & $4.51 \mathrm{~m}$ & & 70.3 & & $3^{\prime \prime \prime}$ & & $4.51 \mathrm{~m}$ & & 70.3 \\
\hline & $4^{\prime \prime \prime}$ & & $4.17 \mathrm{~m}$ & & 68.9 & & $4^{\prime \prime \prime}$ & $4.18 \mathrm{~m}$ & & 68.8 & & $4^{\prime \prime \prime}$ & & $4.16 \mathrm{~m}$ & & 68.9 \\
\hline & $5^{\prime \prime \prime}$ & $\mathrm{a}$ & 4.34 br d & 8.5 & 65.3 & & $5^{\prime \prime \prime}(2 \mathrm{H})$ & $4.18 \mathrm{~m}$ & & 65.3 & & $5^{\prime \prime \prime}$ & $\mathrm{a}$ & 4.31 brd & 10.8 & 65.3 \\
\hline & & $\mathrm{b}$ & 4.16 br d & 8.5 & & & & & & & & & $\mathrm{~b}$ & 4.14 br d & 10.8 & \\
\hline Glc & $1^{\prime \prime \prime \prime \prime}$ & & $6.22 \mathrm{~d}$ & 8.0 & 95.6 & Glc & $1^{\prime \prime \prime \prime}$ & $5.14 \mathrm{~d}$ & 7.8 & 106.8 & Glc & $1^{\prime \prime \prime \prime}$ & & $6.25 \mathrm{~d}$ & 8.1 & 95.6 \\
\hline & $2^{\prime \prime \prime \prime}$ & & $4.09 \mathrm{dd}$ & $8.9,8.0$ & 73.8 & & $2^{\prime \prime \prime \prime}$ & $4.03 \mathrm{dd}$ & $8.6,7.8$ & 75.5 & & $2^{\prime \prime \prime \prime}$ & & $4.08 \mathrm{dd}$ & $9.2,8.1$ & 73.9 \\
\hline & $3^{\prime \prime \prime \prime}$ & & $4.19 \mathrm{dd}$ & $8.9,8.9$ & 78.7 & & $3^{\prime \prime \prime \prime}$ & $4.17 \mathrm{dd}$ & $8.6,8.6$ & 78.8 & & $3^{\prime \prime \prime \prime}$ & & $4.20 \mathrm{dd}$ & $9.2,9.2$ & 78.7 \\
\hline & $4^{\prime \prime \prime \prime}$ & & $4.32 \mathrm{dd}$ & $8.9,8.5$ & 70.8 & & $4^{\prime \prime \prime \prime}$ & $4.24 \mathrm{dd}$ & $8.6,8.6$ & 71.3 & & $4^{\prime \prime \prime \prime \prime}$ & & $4.32 \mathrm{dd}$ & $9.2,8.5$ & 70.8 \\
\hline & $5^{\prime \prime \prime \prime}$ & & $4.06 \mathrm{~m}$ & & 77.9 & & $5^{\prime \prime \prime \prime}$ & $3.89 \mathrm{~m}$ & & 78.8 & & $5^{\prime \prime \prime \prime}$ & & $4.02 \mathrm{~m}$ & & 77.9 \\
\hline & $6^{\prime \prime \prime \prime}$ & $\mathrm{a}$ & 4.63 br d & 11.3 & 69.3 & & $6^{\prime \prime \prime \prime} \quad \mathrm{a}$ & 4.49 br d & 11.5 & 62.6 & & $6^{\prime \prime \prime \prime}$ & $\mathrm{a}$ & 4.65 br d & 11.1 & 69.3 \\
\hline & & $\mathrm{b}$ & 4.28 br d & 11.3 & & & $\mathrm{~b}$ & 4.37 br d & 11.5 & & & & $\mathrm{~b}$ & 4.30 brd & 11.1 & \\
\hline Glc' $^{\prime}$ & $1^{\prime \prime \prime \prime \prime \prime}$ & & $4.90 \mathrm{~d}$ & 7.9 & 105.0 & Glc' & $1^{\prime \prime \prime \prime \prime \prime}$ & $6.26 \mathrm{~d}$ & 8.1 & 95.7 & $\mathrm{Glc}^{\prime}$ & $1^{\prime \prime \prime \prime \prime \prime}$ & & $4.93 \mathrm{~d}$ & 7.9 & 104.7 \\
\hline & $2^{\prime \prime \prime \prime \prime \prime}$ & & $3.89 \mathrm{dd}$ & $8.7,7.9$ & 75.3 & & $2^{\prime \prime \prime \prime \prime \prime}$ & $4.10 \mathrm{dd}$ & $8.7,8.1$ & 73.9 & & $2^{\prime \prime \prime \prime \prime \prime}$ & & $3.91 \mathrm{dd}$ & $8.5,7.9$ & 75.4 \\
\hline & $3^{\prime \prime \prime \prime \prime \prime}$ & & $4.10 \mathrm{dd}$ & $9.4,8.7$ & 76.4 & & $3^{\prime \prime \prime \prime \prime \prime}$ & $4.19 \mathrm{dd}$ & $8.7,8.7$ & 78.6 & & $3^{\prime \prime \prime \prime \prime \prime}$ & & $4.13 \mathrm{dd}$ & $9.3,8.5$ & 76.4 \\
\hline & $4^{\prime \prime \prime \prime \prime \prime}$ & & $4.40 \mathrm{dd}$ & $9.4,9.4$ & 77.2 & & $4^{\prime \prime \prime \prime \prime \prime}$ & $4.33 \mathrm{dd}$ & $8.7,8.7$ & 70.9 & & $4^{\prime \prime \prime \prime \prime}$ & & $4.43 \mathrm{dd}$ & $9.3,9.3$ & 77.2 \\
\hline & $5^{\prime \prime \prime \prime \prime \prime}$ & & $3.53 \mathrm{~m}$ & & 77.0 & & $5^{\prime \prime \prime \prime \prime \prime}$ & $4.08 \mathrm{~m}$ & & 77.9 & & $5^{\prime \prime \prime \prime \prime \prime}$ & & $3.56 \mathrm{~m}$ & & 77.1 \\
\hline & $6^{\prime \prime \prime \prime \prime}$ a & $\mathrm{a}$ & 4.14 br d & 2.5 & 61.2 & & $6^{\prime \prime \prime \prime \prime}$ a & 4.64 br d & 11.1 & 69.3 & & $6^{\prime \prime \prime \prime \prime \prime}$ & $\mathrm{a}$ & 4.15 br d & 11.9 & 61.2 \\
\hline & & $\mathrm{b}$ & 4.00 br d & 12.5 & & & $\mathrm{~b}$ & 4.29 br d & 11.1 & & & & $\mathrm{~b}$ & $4.03 \mathrm{brd}$ & 11.9 & \\
\hline Rha' & $1^{\prime \prime \prime \prime \prime \prime \prime}$ & & 5.84 br s & & 102.4 & $\mathrm{Glc}^{\prime \prime}$ & $1^{\prime \prime \prime \prime \prime \prime \prime}$ & $4.90 \mathrm{~d}$ & 7.9 & 105.1 & Rha' $^{\prime}$ & $1^{\prime \prime \prime \prime \prime \prime \prime}$ & & 5.87 br s & & 102.4 \\
\hline & $2^{\prime \prime \prime \prime \prime \prime \prime}$ & & 4.90 br d & 2.8 & 71.9 & & $2^{\prime \prime \prime \prime \prime \prime \prime}$ & $3.90 \mathrm{dd}$ & $8.9,8.5$ & 75.4 & & $2^{\prime \prime \prime \prime \prime \prime \prime}$ & & $4.90 \mathrm{brd}$ & 2.6 & 71.9 \\
\hline & $3^{\prime \prime \prime \prime \prime \prime \prime}$ & & $4.63 \mathrm{dd}$ & $9.3,2.8$ & 83.8 & & $3^{\prime \prime \prime \prime \prime \prime \prime}$ & $4.11 \mathrm{dd}$ & $9.0,8.9$ & 76.5 & & $3^{\prime \prime \prime \prime \prime \prime \prime}$ & & $4.65 \mathrm{dd}$ & $9.3,2.6$ & 83.9 \\
\hline & $4^{\prime \prime \prime \prime \prime \prime \prime}$ & & $4.50 \mathrm{dd}$ & $9.3,9.3$ & 73.0 & & $4^{\prime \prime \prime \prime \prime \prime \prime}$ & $4.43 \mathrm{dd}$ & $9.0,9.0$ & 77.3 & & $4^{\prime \prime \prime \prime \prime \prime \prime}$ & & $4.43 \mathrm{dd}$ & $9.3,9.3$ & 73.0 \\
\hline & $5^{\prime \prime \prime \prime \prime \prime \prime}$ & & $5.03 \mathrm{dq}$ & $9.3,6.2$ & 70.0 & & $5^{\prime \prime \prime \prime \prime \prime \prime}$ & $3.57 \mathrm{~m}$ & & 77.1 & & $5^{\prime \prime \prime \prime \prime \prime \prime}$ & & $5.04 \mathrm{dq}$ & $9.3,6.2$ & 70.0 \\
\hline & $6^{\prime \prime \prime \prime \prime \prime \prime}$ & & $1.63 \mathrm{~d}$ & 6.2 & 18.4 & & $6^{\prime \prime \prime \prime \prime \prime \prime}$ a & 4.16 br d & 10.5 & 61.2 & & $6^{\prime \prime \prime \prime \prime \prime \prime}$ & & $1.66 \mathrm{~d}$ & 6.2 & 18.4 \\
\hline & & & & & & & $\mathrm{b}$ & 4.03 br d & 10.5 & & & & & & & \\
\hline Glc $^{\prime \prime}$ & $1^{\prime \prime \prime \prime \prime \prime \prime \prime}$ & & $5.34 \mathrm{~d}$ & 7.8 & 106.7 & Rha' $^{\prime}$ & $1^{\prime \prime \prime \prime \prime \prime \prime \prime}$ & 5.88 br s & & 102.5 & Glc $^{\prime \prime}$ & $1^{\prime \prime \prime \prime \prime \prime \prime \prime}$ & & $5.37 \mathrm{~d}$ & 7.8 & 106.8 \\
\hline & $2^{\prime \prime \prime \prime \prime \prime \prime \prime}$ & & $4.07 \mathrm{dd}$ & $8.8,7.8$ & 76.0 & & $2^{\prime \prime \prime \prime \prime \prime \prime \prime}$ & 4.88 br d & 3.0 & 72.0 & & $2^{\prime \prime \prime \prime \prime \prime \prime \prime \prime}$ & & $4.09 \mathrm{dd}$ & $8.8,7.8$ & 76.0 \\
\hline & $3^{\prime \prime \prime \prime \prime \prime \prime \prime}$ & & $4.19 \mathrm{dd}$ & $8.8,8.8$ & 78.4 & & $3^{\prime \prime \prime \prime \prime \prime \prime \prime}$ & $4.67 \mathrm{dd}$ & $9.1,3.0$ & 84.0 & & $3^{\prime \prime \prime \prime \prime \prime \prime \prime}$ & & $4.21 \mathrm{dd}$ & $8.8,8.8$ & 78.4 \\
\hline & $4^{\prime \prime \prime \prime \prime \prime \prime \prime \prime}$ & & $4.26 \mathrm{dd}$ & $8.8,8.5$ & 71.3 & & $4^{\prime \prime \prime \prime \prime \prime \prime \prime}$ & $4.50 \mathrm{dd}$ & $9.6,9.1$ & 73.0 & & $4^{\prime \prime \prime \prime \prime \prime \prime \prime ~}$ & & $4.26 \mathrm{dd}$ & $8.8,8.8$ & 71.3 \\
\hline & $5^{\prime \prime \prime \prime \prime \prime \prime \prime}$ & & $3.83 \mathrm{~m}$ & & 78.4 & & $5^{\prime \prime \prime \prime \prime \prime \prime \prime}$ & $5.06 \mathrm{dq}$ & $9.6,6.1$ & 70.0 & & $5^{\prime \prime \prime \prime \prime \prime \prime \prime}$ & & $3.85 \mathrm{~m}$ & & 78.4 \\
\hline & $6^{\prime \prime \prime \prime \prime \prime \prime \prime} \mathrm{a}$ & & 4.40 br d & 11.2 & 62.3 & & $6^{\prime \prime \prime \prime \prime \prime \prime \prime \prime}$ & $1.67 \mathrm{~d}$ & 6.1 & 18.5 & & $6^{\prime \prime \prime \prime \prime \prime \prime \prime}$ & 'a & 4.41 br d & 11.8 & 62.3 \\
\hline & & $\mathrm{b}$ & 4.34 br d & 11.2 & & & & & & & & & $\mathrm{~b}$ & 4.35 br d & 11.8 & \\
\hline & & & & & & $\mathrm{Glc}^{\prime \prime}$ & $1^{\prime \prime \prime \prime \prime \prime \prime \prime \prime ~}$ & $5.38 \mathrm{~d}$ & 7.8 & 106.8 & & & & & & \\
\hline & & & & & & & $2^{\prime \prime \prime \prime \prime \prime \prime \prime \prime}$ & $4.07 \mathrm{dd}$ & $8.8,7.8$ & 76.1 & & & & & & \\
\hline & & & & & & & $3^{\prime \prime \prime \prime \prime \prime \prime \prime \prime}$ & $4.20 \mathrm{dd}$ & $8.8,8.8$ & 78.4 & & & & & & \\
\hline & & & & & & & $4^{\prime \prime \prime \prime \prime \prime \prime \prime \prime ~}$ & $4.28 \mathrm{dd}$ & $8.8,8.8$ & 71.3 & & & & & & \\
\hline & & & & & & & $5^{\prime \prime \prime \prime \prime \prime \prime \prime \prime \prime ~}$ & $3.86 \mathrm{~m}$ & & 78.4 & & & & & & \\
\hline & & & & & & & $6^{\prime \prime \prime \prime \prime \prime \prime \prime \prime ~} \mathrm{a}$ & $4.41 \mathrm{brd}$ & 11.6 & 62.4 & & & & & & \\
\hline & & & & & & & $\mathrm{b}$ & 4.34 br d & 11.6 & & & & & & & \\
\hline
\end{tabular}

a) Spectra were measured in $\mathrm{C}_{5} \mathrm{D}_{5} \mathrm{~N}$.

sugar chain attached at C-3 of the aglycone moiety was made up of four monosaccharides and differed from that of $\mathbf{1}$ by the presence of an additional glucosyl unit. In a comparison of the ${ }^{13} \mathrm{C}$-NMR spectrum of $\mathbf{2}$ with that of $\mathbf{1}$, a set of six additional signals corresponding to a terminal $\beta$-D-glucopyranosyl unit $(\mathrm{Glc})\left[\delta_{\mathrm{H}-1} 5.14(1 \mathrm{H}, \mathrm{d}, J=7.8 \mathrm{~Hz}) ; \delta_{\mathrm{C}} 106.8,75.5\right.$, $78.8,71.3,78.8$, and 62.6] appeared, and the signal due to $\mathrm{C}$ 4 of the arabinosyl moiety shifted downfield by $11.0 \mathrm{ppm}$ to $\delta 80.4$, suggesting that the C-4 hydroxyl group of the arabi- nosyl moiety is the glycosylated position at which the additional D-glucosyl unit is linked. In the HMBC spectrum of 2 , long-range correlations were observed between H-1 of Rib at $\delta 5.98$ and C-3 of Rha at $\delta 81.1, \mathrm{H}-1$ of Rha at $\delta 6.32$ and C2 of Ara at $\delta 75.8, \mathrm{H}-1$ of Glc at $\delta 5.14$ and C- 4 of Ara at $\delta$ $80.4, \mathrm{H}-1$ of Ara at $\delta 4.72$ and C-3 of the aglycone at $\delta 88.7$, $\mathrm{H}-1$ of Glc'"' at $\delta 5.38$ and C-3 of Rha' at $\delta 84.0, \mathrm{H}-1$ of Rha' at $\delta 5.88$ and C-4 of Glc" at $\delta 77.3, \mathrm{H}-1$ of Glc ${ }^{\prime \prime}$ at $\delta 4.90$ and C-6 of Glc' at $\delta$ 69.3, and between $\mathrm{H}-1$ of Glc' at $\delta 6.26$ 
Table 2. Cytotoxic Activities of Compounds 1-11 against Four Human Cancer Cell Lines ${ }^{a, b)}$

\begin{tabular}{|c|c|c|c|c|}
\hline \multirow{2}{*}{ Compound } & \multicolumn{4}{|c|}{$\mathrm{IC}_{50}(\mu \mathrm{M})$} \\
\hline & HL-60 & A549 & HSC-2 & HSC-4 \\
\hline 4 & $3.1 \pm 0.15$ & $2.9 \pm 0.20$ & $5.9 \pm 0.16$ & $13.0 \pm 0.14$ \\
\hline 5 & $2.3 \pm 0.09$ & $1.5 \pm 0.14$ & $5.7 \pm 0.46$ & $11.7 \pm 0.20$ \\
\hline 6 & $5.7 \pm 0.58$ & $9.6 \pm 0.03$ & $16.3 \pm 0.06$ & $>20$ \\
\hline Oleanolic acid & $>10$ & -c) & - & - \\
\hline Hederagenin & $>10$ & - & - & - \\
\hline Cisplatin $^{d)}$ & $1.6 \pm 0.03$ & $6.4 \pm 0.43$ & $16.9 \pm 0.60$ & $>20$ \\
\hline Etoposide $^{d)}$ & $0.38 \pm 0.02$ & $>20$ & $>20$ & $>20$ \\
\hline Doxorubicin $^{d \text { ) }}$ & $0.07 \pm 0.01$ & $1.7 \pm 0.03$ & $0.25 \pm 0.03$ & $0.88 \pm 0.04$ \\
\hline
\end{tabular}

a) Data represent the mean \pm S.E.M. of three independent experiments. b) Compounds $\mathbf{1}-\mathbf{3}$ and $\mathbf{7}-\mathbf{1 1}$ were not cytotoxic to HL-60 cells (IC $\left.{ }_{50}>10 \mu \mathrm{M}\right)$. c) Not determined. d) Positive control substances.

and $\mathrm{C}-28$ at the aglycone at $\delta 176.5$. Thus, the structure of $\mathbf{2}$ was determined to be $3 \beta$-[(O- $\beta$-D-ribopyranosyl- $(1 \rightarrow 3)-O-\alpha-$ L-rhamnopyranosyl- $(1 \rightarrow 2)$-[ $\beta$-D-glucopyranosyl- $(1 \rightarrow 4)]-\alpha$ L-arabinopyranosyl)oxy]olean-12-en-28-oic acid $O$ - $\beta$-D-glucopyranosyl-( $(1 \rightarrow 3)-O-\alpha$-L-rhamnopyranosyl-( $1 \rightarrow 4)-O-\beta$-Dglucopyranosyl-( $1 \rightarrow 6)-\beta$-D-glucopyranosyl ester.

Compound 3 was shown to have a molecular formula $\mathrm{C}_{70} \mathrm{H}_{114} \mathrm{O}_{35}$ on the basis of HR-ESI-TOF-MS analysis $(\mathrm{m} / \mathrm{z}$ : $\left.1515.7216[\mathrm{M}+\mathrm{H}]^{+}\right)$. Analysis of the ${ }^{1} \mathrm{H}-$ and ${ }^{13} \mathrm{C}-\mathrm{NMR}$ spectra of $\mathbf{3}$ and comparison with that of $\mathbf{1}$ indicated that the triglycoside structure attached to C-3 of the aglycone and the tetraglycoside attached to C-28 are identical to those of $\mathbf{1}$; however, $\mathbf{3}$ slightly differs from $\mathbf{1}$ in terms of the aglycone moiety. The molecular weight of $\mathbf{3}$ was higher than that of $\mathbf{1}$, with the difference corresponding to one oxygen atom. In the ${ }^{13} \mathrm{C}$-NMR spectrum, the signal due to the $\mathrm{C}-23$ methyl group, which was observed at $\delta 28.2$ in 1, appeared as a signal due to a hydroxymethyl carbon at $\delta 64.0$. Furthermore, ABq signals at $\delta 4.30$ and $3.93(J=10.3 \mathrm{~Hz})$ were associated with the hydroxymethyl carbon signal by analysis of the HMQC spectrum. Acid hydrolysis of $\mathbf{3}$ yielded $3 \beta$,23-dihydroxyolean-12en-28-oic acid (hederagenin, 3a), L-rhamnose, D-ribose, Larabinose, and D-glucose. On the basis of these data, the structure of 3 was found to be $3 \beta$-[( $O-\beta$-D-ribopyranosyl$(1 \rightarrow 3)$ - $O$ - $\alpha$-L-rhamnopyranosyl- $(1 \rightarrow 2)-\alpha$-L-arabinopyranosyl)oxy]-23-hydroxyolean-12-en-28-oic acid $O$ - $\beta$-D-glucopyranosyl- $(1 \rightarrow 3)-O-\alpha$-L-rhamnopyranosyl-( $1 \rightarrow 4)-O-\beta$-Dglucopyranosyl-( $(1 \rightarrow 6)-\beta$-D-glucopyranosyl ester.

The isolated compounds $(\mathbf{1}-\mathbf{1 1})$ were evaluated for their cytotoxic activity against HL-60 cells (Table 2). The triterpene monodesmosides $(\mathbf{4}-\mathbf{6})$ showed moderate cytotoxic activity against HL-60 cells with $\mathrm{IC}_{50}$ values of $3.1,2.3$, and $5.7 \mu \mathrm{M}$, respectively. Oleanolic acid (the aglycone of $\mathbf{4}$ and 5), hederagenin (the aglycone of 6), and bisdesmosides with the sugar units at both the C-3 hydroxy group and C-28 carboxy group (1-3 and 7-11) did not exhibit cytotoxicity even at a sample concentration of $10 \mu \mathrm{M}$. Compounds $4-6$ also exhibited cytotoxic activities against A459 and HSC-2 solid tumor cells greater than or equal to those of cisplatin, which was used as a positive control. A459 and HSC-2 cells were not sensitive to etoposide. Compounds $\mathbf{4}$ and $\mathbf{5}$ were moderately cytotoxic to HSC-4 cells, which were resistant to both cisplatin and etoposide. Compound $\mathbf{6}$ is the C-23 hydroxy derivative of $\mathbf{4}$, and it was presumed that the hydroxy group in $\mathbf{6}$ diminishes its cytotoxicity against HSC-4 cells.

\section{Experimental}

Optical rotations were measured using a JASCO P-1030 (Tokyo, Japan) automatic digital polarimeter. IR spectra were recorded on a JASCO FT-IR 620 spectrophotometer. NMR spectra were recorded on a Bruker DRX-500 (500 MHz for ${ }^{1} \mathrm{H}-\mathrm{NMR}$, Karlsruhe, Germany) and a Bruker DRX-600 (600 MHz for $\left.{ }^{1} \mathrm{H}-\mathrm{NMR}\right)$ spectrometer using standard Bruker pulse programs. 1D-TOCSY and HSQC-TOCSY spectra were recorded at the mixing time of $120 \mathrm{~ms}$. Chemical shifts are given as $\delta$ values in reference to tetramethylsilane (TMS) as an internal standard. ESI-TOF-MS spectra were recorded on a Waters-Micromass LCT mass spectrometer (Manchester, U.K.). Diaion HP-20 (Mitsubishi Chemical, Tokyo, Japan), silica gel (Fuji Silysia Chemical, Aichi, Japan), and ODS silica gel (Nacalai Tesque, Kyoto, Japan) were used for column chromatography. TLC was carried out on Silica gel $60 \mathrm{~F}_{254}$ (thickness: $0.25 \mathrm{~mm}$, Merck, Darmstadt, Germany) and $\mathrm{RP}_{18}$ $\mathrm{F}_{254 \mathrm{~s}}$ plates (thickness: $0.25 \mathrm{~mm}$, Merck), and spots were visualized by spraying the plates with $10 \% \mathrm{H}_{2} \mathrm{SO}_{4}$ solution, followed by heating. HPLC was performed by using a system composed of a CCPM pump (Tosoh, Tokyo, Japan), a PX-8010 controller (Tosoh), an RI-8010 (Tosoh) and a Shodex OR-2 (Showa Denko, Tokyo, Japan) detector, and a Rheodyne injection port. The following reagents were obtained from the indicated companies: RPMI 1640, minimum essential medium (MEM), and 3-(4,5-dimethylthiazol-2-yl)-2,5-diphenyl-2H-tetrazolium bromide (MTT) (SigmaAldrich, St. Louis, MO, U.S.A.); fetal bovine serum (FBS) (BioWhittaker, Walkersville, MO, U.S.A.); Dulbecco's modified Eagle's medium (DMEM), penicillin G sodium salt, and streptomycin sulfate (Gibco, Grand Island, NY, U.S.A.). All other chemicals used were of biochemical reagent grade.

Plant Material The young plants of Anemone hupehensis var. japonica were purchased from Sakata Seed Co., Ltd. (Kanagawa, Japan) in June 2002. The plants were cultivated in the botanical garden of Tokyo University of Pharmacy and Life Sciences, and identified by Dr. Yutaka Sashida, emeritus professor of medicinal pharmacognosy at Tokyo University of Pharmacy and Life Sciences. A voucher specimen has been deposited in our laboratory (voucher No. AH-2003-001, Laboratory of Medicinal Pharmacognosy).

Extraction and Isolation The plant material (dry weight, $3.3 \mathrm{~kg}$ ) was extracted with hot $\mathrm{MeOH}$ (261). The $\mathrm{MeOH}$ extract was concentrated under reduced pressure, and the viscous concentrate $(180 \mathrm{~g})$ was passed through a Diaion HP-20 column ( $85 \mathrm{~mm}$ inner diameter (i.d.). $\times 200 \mathrm{~mm}$ ) that was successively eluted with $30 \% \mathrm{MeOH}, 50 \% \mathrm{MeOH}, \mathrm{MeOH}$, EtOH, and EtOAc (each 71). Column chromatography of the $\mathrm{MeOH}$ eluate portion $(50 \mathrm{~g})$ on silica gel $(70 \mathrm{~mm}$ i.d. $\times 330 \mathrm{~mm})$ and elution with a stepwise gradient mixture of $\mathrm{CHCl}_{3}-\mathrm{MeOH}-\mathrm{H}_{2} \mathrm{O}(9: 1: 0 ; 30: 10: 1 ; 20: 10: 1 ; 7: 4: 1)$, and finally with $\mathrm{MeOH}$ alone, yielded eight fractions $(\mathrm{A}-\mathrm{H})$. Fraction D was chromatographed on an ODS silica gel column $(60 \mathrm{~mm}$ i.d. $\times 240 \mathrm{~mm})$ eluted with $\mathrm{MeOH}-\mathrm{H}_{2} \mathrm{O}(4: 1)$ to give 14 subfractions (D-1-D-14). Fraction D-7 was suspended in $\mathrm{MeOH}$ and the insoluble solid was filtrated to give 6 $(1.00 \mathrm{~g})$. Fraction D-11 was chromatographed on silica gel $(25 \mathrm{~mm}$ i.d. $\times 200 \mathrm{~mm})$ eluted with $\mathrm{CHCl}_{3}-\mathrm{MeOH}-\mathrm{H}_{2} \mathrm{O}(50: 10: 1)$ to give $4(15.1$ $\mathrm{mg})$. Fraction D-12 was chromatographed on silica gel $(35 \mathrm{~mm}$ i.d. $\times 200$ $\mathrm{mm})$ eluted with $\mathrm{CHCl}_{3}-\mathrm{MeOH}-\mathrm{H}_{2} \mathrm{O}(70: 10: 1)$ to give 5 (137 mg). Fraction $\mathrm{E}$ was chromatographed on an ODS silica gel column $(40 \mathrm{~mm}$ i.d. $\times 220 \mathrm{~mm}$ ) eluted with $\mathrm{MeOH}-\mathrm{H}_{2} \mathrm{O}(7: 3)$ to give seven subfractions (E$1-\mathrm{E}-7)$. Fraction E-3 was subjected to column chromatography on silica gel $(35 \mathrm{~mm}$ i.d. $\times 200 \mathrm{~mm})$ eluted with $\mathrm{CHCl}_{3}-\mathrm{MeOH}-\mathrm{H}_{2} \mathrm{O}(30: 10: 1 ; 20: 10$ : $1)$ and on ODS silica gel $(25 \mathrm{~mm}$ i.d. $\times 240 \mathrm{~mm})$ eluted with $\mathrm{MeOH}-\mathrm{H}_{2} \mathrm{O}$ $(2: 1)$ to give $9(6.2 \mathrm{mg})$. Fraction E-4 was subjected to column chromatography on silica gel $(35 \mathrm{~mm}$ i.d. $\times 200 \mathrm{~mm})$ eluted with $\mathrm{CHCl}_{3}-\mathrm{MeOH}-\mathrm{H}_{2} \mathrm{O}$ 
$(30: 10: 1)$ and on ODS silica gel $(25 \mathrm{~mm}$ i.d. $\times 250 \mathrm{~mm})$ eluted with $\mathrm{MeOH}-\mathrm{H}_{2} \mathrm{O}(7: 3)$ to give $7(17.6 \mathrm{mg})$. Fraction F was separated by an ODS silica gel column $(40 \mathrm{~mm}$ i.d. $\times 240 \mathrm{~mm})$ eluted with $\mathrm{MeOH}-\mathrm{H}_{2} \mathrm{O}(7: 3)$ to give $8(371 \mathrm{mg})$. Fraction $\mathrm{G}$ was subjected to silica gel column chromatography $(60 \mathrm{~mm}$ i.d. $\times 250 \mathrm{~mm})$ eluted with $\mathrm{CHCl}_{3}-\mathrm{MeOH}-\mathrm{H}_{2} \mathrm{O}(20: 10: 1)$ and ODS silica gel column chromatography $(40 \mathrm{~mm}$ i.d. $\times 240 \mathrm{~mm})$ eluted with $\mathrm{MeOH}-\mathrm{H}_{2} \mathrm{O}(7: 3)$ to give $\mathbf{1 0}(527 \mathrm{mg})$. Fraction $\mathrm{H}$ was chromatographed on an ODS silica gel column $(60 \mathrm{~mm}$ i.d. $\times 300 \mathrm{~mm})$ eluted with $\mathrm{MeOH}-\mathrm{H}_{2} \mathrm{O}$ ( $7: 3)$ to give 12 subfractions $(\mathrm{H}-1-\mathrm{H}-12)$. Fraction $\mathrm{H}-3$ was subjected to column chromatography on ODS silica gel $(40 \mathrm{~mm}$ i.d. $\times 220 \mathrm{~mm})$ eluted with $\mathrm{MeOH}-\mathrm{H}_{2} \mathrm{O}(3: 2)$ and on silica gel $(25 \mathrm{~mm}$ i.d. $\times 270 \mathrm{~mm})$ eluted with $\mathrm{CHCl}_{3}-\mathrm{MeOH}-\mathrm{H}_{2} \mathrm{O}(7: 4: 1)$ to give $11(37.8 \mathrm{mg})$. Fraction $\mathrm{H}-4$ was chromatographed on silica gel $(40 \mathrm{~mm}$ i.d. $\times 250 \mathrm{~mm})$ eluted with $\mathrm{CHCl}_{3}$ $\mathrm{MeOH}-\mathrm{H}_{2} \mathrm{O}(20: 10: 1)$ and on ODS silica gel $(25 \mathrm{~mm}$ i.d. $\times 240 \mathrm{~mm})$ eluted with $\mathrm{MeOH}-\mathrm{H}_{2} \mathrm{O}(3: 2)$ to give $3(35.3 \mathrm{mg})$. Fraction $\mathrm{H}-6$ was subjected to column chromatography on silica gel $(35 \mathrm{~mm}$ i.d. $\times 230 \mathrm{~mm})$ eluted with $\mathrm{CHCl}_{3}-\mathrm{MeOH}-\mathrm{H}_{2} \mathrm{O}(20: 10: 1)$ and on ODS silica gel $(25 \mathrm{~mm}$ i.d. $\times 230$ $\mathrm{mm})$ eluted with $\mathrm{MeOH}-\mathrm{H}_{2} \mathrm{O}(2: 1)$ to give $2(6.7 \mathrm{mg})$. Fraction $\mathrm{H}-9$ was chromatographed on silica gel $(20 \mathrm{~mm}$ i.d. $\times 220 \mathrm{~mm})$ eluted with $\mathrm{CHCl}_{3}$ $\mathrm{MeOH}-\mathrm{H}_{2} \mathrm{O}(20: 10: 1)$ to give $1(45.3 \mathrm{mg})$.

Compound 1: Amorphous solid. $[\alpha]_{\mathrm{D}}^{25}-33.6^{\circ}(c=0.10, \mathrm{MeOH})$. HR-ESITOF-MS $m / z$ : $1521.7109[\mathrm{M}+\mathrm{Na}]^{+}$(Calcd for $\left.\mathrm{C}_{70} \mathrm{H}_{114} \mathrm{O}_{34} \mathrm{Na}: 1521.7089\right)$. IR $v_{\max }($ film $) \mathrm{cm}^{-1}: 3376(\mathrm{OH}), 2925(\mathrm{CH}), 1740(\mathrm{C}=\mathrm{O}), 1260$ and 1064 $(\mathrm{C}-\mathrm{O}) .{ }^{1} \mathrm{H}-\mathrm{NMR}\left(500 \mathrm{MHz}, \mathrm{C}_{5} \mathrm{D}_{5} \mathrm{~N}\right) \delta: 5.39(1 \mathrm{H}$, br s, H-12), $3.26(1 \mathrm{H}, \mathrm{dd}$ $J=11.5,3.8 \mathrm{~Hz}, \mathrm{H}-3), 3.17$ (1H, dd, $J=10.1,3.3 \mathrm{~Hz}, \mathrm{H}-18), 1.28(3 \mathrm{H}, \mathrm{s}, \mathrm{Me}-$ 23), 1.23 (3H, s, Me-27), 1.13 (3H, s, Me-24), 1.06 (3H, s, Me-26), 0.89 $(3 \mathrm{H}, \mathrm{s}, \mathrm{Me}-29), 0.87$ (3H, s, Me-30), 0.85 (3H, s, Me-25); for signals of sugar moieties, see Table $1 .{ }^{13} \mathrm{C}-\mathrm{NMR}\left(125 \mathrm{MHz}, \mathrm{C}_{5} \mathrm{D}_{5} \mathrm{~N}\right) \delta: 38.9(\mathrm{C}-1)$, 26.6 (C-2), 88.7 (C-3), 39.6 (C-4), 56.0 (C-5), 18.5 (C-6), 33.1 (C-7), 39.9 (C-8), 48.0 (C-9), 37.0 (C-10), 23.8 (C-11), 122.8 (C-12), 144.1 (C-13), 42.1 (C-14), 28.3 (C-15), 23.3 (C-16), 47.0 (C-17), 41.7 (C-18), 46.2 (C-19), 30.7 (C-20), 34.0 (C-21), 32.5 (C-22), 28.2 (C-23), 17.1 (C-24), 15.6 (C-25), 17.4 (C-26), 26.1 (C-27), 176.5 (C-28), 33.1 (C-29), 23.7 (C-30); for signals for the sugar moieties, see Table 1 .

Acid Hydrolysis of $\mathbf{1}$ A solution of $\mathbf{1}(12.0 \mathrm{mg})$ in $1 \mathrm{M} \mathrm{HCl}$ (dioxane$\mathrm{H}_{2} \mathrm{O}, 1: 1,3 \mathrm{ml}$ ) was heated at $95^{\circ} \mathrm{C}$ for $1.5 \mathrm{~h}$ under an $\mathrm{Ar}$ atmosphere. After cooling, the reaction mixture was neutralized by passage through an Amberlite IRA-96SB (Organo, Tokyo, Japan) column and chromatographed on Diaion $\mathrm{HP}-20$ eluted with $\mathrm{MeOH}-\mathrm{H}_{2} \mathrm{O}(2: 3)$ followed by $\mathrm{EtOH}-\mathrm{Me}_{2} \mathrm{CO}$ $(1: 1)$, to yield an aglycone fraction $(3.7 \mathrm{mg})$ and a sugar fraction $(8.6 \mathrm{mg})$ The aglycone fraction was chromatographed on silica gel eluted with hexane- $\mathrm{Me}_{2} \mathrm{CO}(4: 1)$ to give $\mathbf{1 a}$ (oleanolic acid, $2.3 \mathrm{mg}$ ). The sugar fraction was analyzed by HPLC under the following conditions: column, Capcell Pak NH2 UG80 (4.6 mm i.d. $\times 250 \mathrm{~mm}, 5 \mu \mathrm{m}$, Shiseido, Tokyo, Japan); solvent, $\mathrm{MeCN}-\mathrm{H}_{2} \mathrm{O}(17: 3)$; flow rate, $0.9 \mathrm{ml} / \mathrm{min}$; detection, refractive index (RI) and optical rotation $(\mathrm{OR})$. Identification of L-rhamnose, D-ribose, D-arabinose, and D-glucose present in the sugar fraction was carried out by comparison of their retention times $\left(t_{\mathrm{R}}\right)$ and optical rotations with those of authentic samples. $t_{\mathrm{R}}(\mathrm{min})$ : 7.6 (L-rhamnose, negative optical rotation), 8.2 (D-ribose, negative optical rotation), 9.0 (D-arabinose, positive optical rotation), 15.6 (D-glucose, positive optical rotation).

Compound 2: Amorphous solid. $[\alpha]_{\mathrm{D}}^{25}-20.2^{\circ}(c=0.10, \mathrm{MeOH})$. HR-ESITOF-MS $m / z: 1661.7778[\mathrm{M}+\mathrm{H}]^{+}$(Calcd for $\left.\mathrm{C}_{76} \mathrm{H}_{125} \mathrm{O}_{39}: 1661.7798\right)$. IR $v_{\max }($ film $) \mathrm{cm}^{-1}$ : $3376(\mathrm{OH}), 2925(\mathrm{CH}), 1739(\mathrm{C}=\mathrm{O}), 1260,1092$ and $1027(\mathrm{C}-\mathrm{O}) .{ }^{1} \mathrm{H}-\mathrm{NMR}\left(500 \mathrm{MHz}, \mathrm{C}_{5} \mathrm{D}_{5} \mathrm{~N}\right) \delta: 5.42(1 \mathrm{H}$, br s, H-12) 3.25 $(1 \mathrm{H}, \mathrm{dd}, J=11.5,3.8 \mathrm{~Hz}, \mathrm{H}-3), 3.19(1 \mathrm{H}, \mathrm{dd}, J=13.4,3.4 \mathrm{~Hz}, \mathrm{H}-18), 1.31$ (3H, s, Me-23), 1.26 (3H, s, Me-27), 1.18 (3H, s, Me-24), 1.09 (3H, s, Me26), $0.92(3 \mathrm{H}, \mathrm{s}, \mathrm{Me}-29), 0.90(3 \mathrm{H}, \mathrm{s}, \mathrm{Me}-30), 0.88(3 \mathrm{H}, \mathrm{s}, \mathrm{Me}-25)$; for signals of sugar moieties, see Table $1 .{ }^{13} \mathrm{C}-\mathrm{NMR}\left(125 \mathrm{MHz}, \mathrm{C}_{5} \mathrm{D}_{5} \mathrm{~N}\right) \delta: 39.0(\mathrm{C}$ 1), 26.7 (C-2), 88.7 (C-3), 39.6 (C-4), 56.1 (C-5), 18.5 (C-6), 33.2 (C-7), 39.9 (C-8), 48.1 (C-9), 37.0 (C-10), 23.7 (C-11), 123.1 (C-12), 144.1 (C-13), 42.2 (C-14), 28.2 (C-15), 23.4 (C-16), 47.0 (C-17), 41.7 (C-18), 46.3 (C-19), 30.8 (C-20), 34.0 (C-21), 32.5 (C-22), 28.2 (C-23), 17.2 (C-24), 15.7 (C-25), 17.5 (C-26), 26.1 (C-27), 176.5 (C-28), 33.2 (C-29), 23.7 (C-30); for signals of sugar moieties, see Table 1 .

Acid Hydrolysis of 2 A solution of $\mathbf{2}(6.0 \mathrm{mg})$ was subjected to acid hydrolysis using the above-mentioned procedure for the hydrolysis of $\mathbf{1}$ to afford an aglycone fraction $(1.5 \mathrm{mg})$ and a sugar fraction $(4.5 \mathrm{mg})$. The aglycone fraction was chromatographed on silica gel eluted with hexane- $\mathrm{Me}_{2} \mathrm{CO}$ $(4: 1)$ to give 1 a $(0.8 \mathrm{mg})$. HPLC analysis of the sugar fraction under the same conditions as in the analysis of $\mathbf{1}$ showed the presence of L-rhamnose, D-ribose, D-arabinose, and D-glucose. $t_{\mathrm{R}}(\mathrm{min})$ : 7.7 (L-rhamnose, negative optical rotation), 8.3 (D-ribose, negative optical rotation), 9.7 (D-arabinose, positive optical rotation), 17.2 (D-glucose, positive optical rotation).
Compound 3: Amorphous solid. $[\alpha]_{\mathrm{D}}^{25}-29.3^{\circ}(c=0.10, \mathrm{MeOH})$. HR-ESITOF-MS $m / z: 1515.7216[\mathrm{M}+\mathrm{H}]^{+}$(Calcd for $\left.\mathrm{C}_{70} \mathrm{H}_{115} \mathrm{O}_{35}: 1515.7219\right)$. IR $v_{\max }($ film $) \mathrm{cm}^{-1}: 3376(\mathrm{OH}), 2936(\mathrm{CH}), 1732(\mathrm{C}=\mathrm{O}), 1260$ and $1061(\mathrm{C}-$ O). ${ }^{1} \mathrm{H}-\mathrm{NMR}\left(500 \mathrm{MHz}, \mathrm{C}_{5} \mathrm{D}_{5} \mathrm{~N}\right) \delta: 5.41(1 \mathrm{H}, \mathrm{brs}, \mathrm{H}-12), 4.29(1 \mathrm{H}, \mathrm{m}, \mathrm{H}-$ 3), 4.30 (1H, d, $J=10.3 \mathrm{~Hz}, \mathrm{H}-23 \mathrm{a}), 3.93$ (1H, d, $J=10.3 \mathrm{~Hz}, \mathrm{H}-23 \mathrm{~b}), 3.17$ (1H, dd, $J=13.4,3.3 \mathrm{~Hz}, \mathrm{H}-18), 1.19$ (3H, s, Me-27), 1.14 (3H, s, Me-24), $1.11(3 \mathrm{H}, \mathrm{s}, \mathrm{Me}-26), 0.97$ (3H, s, Me-25), $0.88(3 \mathrm{H} \times 2, \mathrm{~s}, \mathrm{Me}-29$ and Me$30)$; for signals of sugar moieties, see Table $1 .{ }^{13} \mathrm{C}-\mathrm{NMR}\left(125 \mathrm{MHz}, \mathrm{C}_{5} \mathrm{D}_{5} \mathrm{~N}\right)$ $\delta: 39.1(\mathrm{C}-1), 26.4(\mathrm{C}-2), 81.1$ (C-3), $43.6(\mathrm{C}-4), 47.7$ (C-5), 18.1 (C-6), 32.8 (C-7), 39.9 (C-8), 48.2 (C-9), 36.9 (C-10), 23.8 (C-11), 123.0 (C-12), 144.1 (C-13), 42.1 (C-14), 28.3 (C-15), 23.3 (C-16), 47.0 (C-17), 41.6 (C18), 46.2 (C-19), 30.7 (C-20), 34.0 (C-21), 32.5 (C-22), 64.0 (C-23), 14.1 (C-24), 16.2 (C-25), 17.5 (C-26), 26.0 (C-27), 176.5 (C-28), 33.1 (C-29), 23.7 (C-30); for signals of sugar moieties, see Table 1

Acid Hydrolysis of 3 A solution of $3(12.0 \mathrm{mg})$ was subjected to acid hydrolysis using the above-mentioned procedure for the hydrolysis of $\mathbf{1}$ to afford an aglycone fraction $(3.3 \mathrm{mg}$ ) and a sugar fraction $(7.4 \mathrm{mg})$. The aglycone fraction was chromatographed on silica gel eluted with hexane- $\mathrm{Me}_{2} \mathrm{CO}$ $(4: 1)$ to give $\mathbf{3 a}$ (hederagenin, $1.7 \mathrm{mg}$ ). HPLC analysis of the sugar fraction under the same conditions as in the analysis of $\mathbf{1}$ showed the presence of $\mathrm{L}$ rhamnose, D-ribose, D-arabinose, and D-glucose. $t_{\mathrm{R}}(\mathrm{min})$ : 7.6 (L-rhamnose, negative optical rotation), 8.2 (D-ribose, negative optical rotation), 9.0 (Darabinose, positive optical rotation), 15.6 (D-glucose, positive optical rotation).

Cell Culture and Assay for Cytotoxic Activity against HL-60 Cells HL-60 cells, which were obtained from the Human Science Research Resources Bank (JCRB 0085, Osaka, Japan) were maintained in RPMI 1640 medium containing $10 \%$ heat-inactivated FBS and antibiotics (100 units $/ \mathrm{ml}$ penicillin sodium salt and $100 \mu \mathrm{g} / \mathrm{ml}$ streptomycin sulfate) in a $5 \% \mathrm{CO}_{2}$ humidified incubator at $37^{\circ} \mathrm{C}$. The cells were washed and resuspended in the medium at $4 \times 10^{4}$ cells $/ \mathrm{ml}$, and $196 \mu$ of this cell suspension was seeded into 96-well flat bottom plates (Iwaki Glass, Chiba, Japan). The cells were incubated in $5 \% \mathrm{CO}_{2} /$ air for $24 \mathrm{~h}$ at $37^{\circ} \mathrm{C}$. After incubation, $4 \mu \mathrm{l}$ of EtOH $-\mathrm{H}_{2} \mathrm{O}(1: 1)$ solution containing the sample was added to obtain final concentrations of $0.01-20 \mu \mathrm{M}$, and $4 \mu \mathrm{l}$ of EtOH- $\mathrm{H}_{2} \mathrm{O}(1: 1)$ was added to the control wells. The cells were further incubated for $72 \mathrm{~h}$ in the presence of each agent, and then cell growth was evaluated using a modified MTT reduction assay. ${ }^{14)}$ At the end of the incubation period, $10 \mu \mathrm{l}$ of $5 \mathrm{mg} / \mathrm{ml}$ MTT in phosphate buffered saline (PBS) was added to each well, and the plate was further incubated in $5 \% \mathrm{CO}_{2} /$ air for $4 \mathrm{~h}$ at $37^{\circ} \mathrm{C}$. Then, the plate was centrifuged at $1500 \boldsymbol{g}$ for $5 \mathrm{~min}$ to precipitate MTT formazan. An aliquot of $150 \mu \mathrm{l}$ of the supernatant was removed from each well, and $175 \mu \mathrm{l}$ of dimethylsulfoxide (DMSO) was added to dissolve the MTT formazan crystals. The plate was mixed on a microplate mixer for $10 \mathrm{~min}$ and then read on a microplate reader (Spectra Classic, Tecan, Salzburg, Austria) at $550 \mathrm{~nm}$. Each assay was performed in triplicate and cytotoxicity was expressed as the $\mathrm{IC}_{50}$ value, which reduces the number of viable cells by $50 \%$.

Cell Culture and Assay for Cytotoxic Activity against A549, HSC-2, and HSC-4 Cells A549 (JCRB 0076) cells were incubated at $37^{\circ} \mathrm{C}$ in MEM supplemented with $10 \%$ heat-inactivated FBS in a humidified $5 \%$ $\mathrm{CO}_{2}$ atmosphere. HSC-2 and HSC-4 cells were maintained as monolayer cultures at $37^{\circ} \mathrm{C}$ in DMEM supplemented with $10 \%$ heat-inactivated FBS in a humidified $5 \% \mathrm{CO}_{2}$ atmosphere. Cells were trypsinized and resuspended in the medium at $1 \times 10^{5}$ cells $/ \mathrm{ml}$, and $100 \mu \mathrm{l}$ of this cell suspension was seeded into 96-well flat bottom plates and incubated for $24 \mathrm{~h}$. After washing once with PBS, the cells were treated for $24 \mathrm{~h}$ in the presence of the test compounds. The cells were washed once with PBS and incubated for $4 \mathrm{~h}$ with $0.2 \mathrm{mg} / \mathrm{ml} \mathrm{MTT} \mathrm{in} \mathrm{MEM} \mathrm{(A549} \mathrm{cells)} \mathrm{or} \mathrm{DMEM} \mathrm{(HSC-2} \mathrm{and} \mathrm{HSC-4}$ cells), supplemented with $10 \%$ heat-inactivated FBS. After the medium was removed, the MTT formazan crystals were dissolved with $100 \mu \mathrm{l}$ of DMSO. The plate was mixed on a microplate mixer for $10 \mathrm{~min}$ and then read on a microplate reader at $550 \mathrm{~nm}$. Each assay was performed in triplicate and cytotoxicity was expressed as the $\mathrm{IC}_{50}$ value, which reduces the number of viable cells by $50 \%$.

\section{References and Notes}

1) "The Grand Dictionary of Horticulture," Vol. 1, ed. by Tsukamoto Y., Shogakukan, Tokyo, 1989, pp. 109-110.

2) Jiangsu New Medical College, "The Dictionary of Chinese Medicinal Materials," Vol. 1, Shanghai Scientific and Technological Press, Shanghai, 1977, pp. 640-641.

3) Wang M. K., Chen Y. Z., Wu F. E., Huaxue Xuebao, 52, 609-612 (1994).

4) Mizutani K., Ohtani K., Wei J. X., Kasai R., Tanaka O., Planta Med., 
50, 327-331 (1984)

5) Dubois M. A., Ilyas M., Wagner H., Planta Med., 52, 80-83 (1986).

6) Dovgii I. I., Grishkovets V. I., Kachala V. V., Shashkov A. S., Chem. Nat. Compd., 42, 182-185 (2006).

7) Wang M., Wu F., Chen Y., Phytochemistry, 44, 333-335 (1997).

8) Shao B., Qin G., Xu R., Wu H., Ma K., Phytochemistry, 38, 1473 1479 (1995).

9) Yokosuka A., Mimaki Y., Sakuma C., Sashida Y., Steroids, 70, 257265 (2005).
10) Yokosuka A., Mimaki Y., Chem. Pharm. Bull., 52, 1396-1398 (2004).

11) Yokosuka A., Kawakami S., Haraguchi M., Mimaki Y., Tetrahedron, 64, 1474-1481 (2008).

12) Agrawal P. K., Jain D. C., Gupta R. K., Thakur R. S., Phytochemistry, 24, 2479-2496 (1985).

13) Agrawal P. K., Phytochemistry, 31, 3307-3330 (1992).

14) Sargent J. M., Taylor C. G., Br. J. Cancer, 60, 206-210 (1989). 